\title{
Global migration of impurities in tokamaks
}

A Hakola ${ }^{1} \ddagger$, M I Airila ${ }^{1}$, C Björkas ${ }^{2}$, D Borodin $^{3}$, S Brezinsek ${ }^{3}$, J P Coad ${ }^{1}$, M Groth ${ }^{4}$, A Järvinen ${ }^{4}$, A Kirschner ${ }^{3}$, S

Koivuranta $^{1}$, K Krieger ${ }^{5}$, T Kurki-Suonio ${ }^{4}$, J Likonen ${ }^{1}$, V

Lindholm $^{4}$, T Makkonen ${ }^{4}$, M Mayer $^{5}$, J Miettunen ${ }^{4}$, H W

Müller $^{5}, \mathbf{R ~ N e u ^ { 5 } , \text { P Petersson }}{ }^{6}$, V Rohde ${ }^{5}, \mathrm{M} \mathrm{Rubel}^{6}$, A

Widdowson ${ }^{7}$, the ASDEX Upgrade Team, JET-EFDA

Contributors $\S$

${ }^{1}$ VTT Technical Research Centre of Finland, Association EURATOM-Tekes, P.O. Box 1000, FI-02044 VTT, Finland

${ }^{2}$ University of Helsinki, Department of Physics, Association EURATOM-Tekes, P.O.

Box 43, FI-00014 University of Helsinki, Finland

${ }^{3}$ Institute of Energy and Climate Research - Plasma Physics, Forschungszentrum Jülich GmbH, Association EURATOM-FZJ, D-52425 Jülich, Germany

${ }^{4}$ Aalto University, Department of Applied Physics, Association EURATOM-Tekes,

P. O. Box 14100, FI-00076 AALTO, Finland

${ }^{5}$ Max-Planck-Institut für Plasmaphysik, EURATOM Association, Boltzmannstrasse

2, D-85748 Garching, Germany

${ }^{6}$ Royal Institute of Technology, Association EURATOM-VR, SE-10044 Stockholm, Sweden

${ }^{7}$ EURATOM/CCFE Fusion Association, Culham Science Centre, Abingdon, Oxon OX14 3DB, UK

E-mail: antti.hakola@vtt.fi

\begin{abstract}
Migration of impurities in tokamaks has been studied with the help of tracer-injection $\left({ }^{13} \mathrm{C}\right.$ and $\left.{ }^{15} \mathrm{~N}\right)$ experiments in JET and ASDEX Upgrade since 2001. We have identified a common pattern for the migrating particles: SOL flows drive impurities from the low-field side towards the high-field side of the vessel. Migration is also sensitive to the density and magnetic configuration of the plasma, and strong local variations in the resulting deposition patterns require $3 \mathrm{D}$ treatment of the migration process. Moreover, re-erosion of the deposited particles has to be taken into account to properly describe the migration process during steady-state operations of the reactor.
\end{abstract}

PACS numbers: 52.25.Vy, 52.40.Hf, 52.55.Fa, 52.65.-y, 82.80.Ms

Submitted to: Plasma Phys. Control. Fusion

$\ddagger$ Corresponding author.

$\S$ See App. of F. Romanelli et al., Proc. of the 24th IAEA Fusion Energy Conf. 2012, San Diego, the United States 


\section{Introduction}

Erosion of plasma-facing components (PFCs) is a critical issue in future fusion reactors. This is not only because the lifetime of PFCs can be severely restricted but also because the eroded material can migrate in the scrape-off layer (SOL) plasma and form thick co-deposited layers with the plasma and impurity particles [1]. If located in places hard to access, such layers cannot be easily removed, which may lead to unacceptably high inventories of tritium in the reactor vessel - after only a short period of plasma operations [2]. In ITER, an additional concern is the formation of mixed material layers whose thermo-mechanical properties may be inferior to those of pure PFC materials [3]. To guarantee successful operation of a reactor, it is therefore important to form an adequate picture of various migration mechanisms and the underlying physics.

This article concentrates on the migration of impurities in tokamak-type fusion reactors. We have investigated the effect of different plasma parameters, operational regimes, wall material, and the impurity element on the global deposition patterns and study how different these patterns are when the source of impurities is located in the main chamber - mainly in the low-field side (outer) midplane - or in the outer divertor. These two regions are at the focus of our studies since they are known to be strong net-erosion zones in tokamaks [4-6]. In addition to obtaining an extensive experimental database, we aim at interpreting the results such that the migration pathways of different elements, e.g., in ITER could be predicted.

The simplest way to investigate migration processes is to use specific marker probes as PFCs, expose them to plasma discharges during a pre-determined period, and measure the erosion of the markers and the deposition of the eroded material on closely-lying wall structures. While this method allows determining net erosion and deposition, the results are often integrated over several plasma scenarios, thus making full modelling of the experiment next to impossible. Moreover, in practice only a few toroidal and poloidal locations of a tokamak can be covered using marker probes.

An alternative strategy is to introduce a tracer element into the tokamak from a pre-defined location during identical, well-characterized discharges, and determine its deposition profile on various PFCs immediately after the experiment [7]. The natural abundance of the tracer should be small $(<1-2$ at.\%), it should behave similarly to the main impurity, and it should be easily detectable by standard surface analysis techniques. Good candidates for tracers have proven to be ${ }^{13} \mathrm{C}[7]$ and ${ }^{15} \mathrm{~N}[8]$ but also $\mathrm{Si}$ (from $\mathrm{SiD}_{4}$ ) and $\mathrm{W}$ (from $\mathrm{WF}_{6}$ ) have been used in experiments, e.g., in TEXTOR, having carbon PFCs [9].

This article gives first a brief summary of the tracer-injection experiments carried out in different tokamaks, with the emphasis put on global results obtained in JET and ASDEX Upgrade (AUG). In Chapter 3, two different cases - migration of impurities originating from a main-chamber source (AUG) and from a divertor source (JET) are discussed and the pieces of physics needed to reproduce the experimental results are discussed. Finally, Chapter 4 concludes the paper. 
Table 1. Main parameters of the different global injection experiments at JET and AUG. Here, LSN=lower single null, USN=upper single null, DN=double null, $\mathrm{OD}=$ outer divertor, $\mathrm{OMP}=$ outer midplane, $\mathrm{CFC}=$ carbon fibre composite.

\begin{tabular}{|l|l|l|l|l|l|l|}
\hline Experiment & $\begin{array}{l}\text { Injection } \\
\text { source }\end{array}$ & $\begin{array}{l}\text { Type of } \\
\text { discharges }\end{array}$ & $\begin{array}{l}\text { Plasma } \\
\text { gas }\end{array}$ & $\begin{array}{l}\text { Density } \\
\left(\times 10^{19} \mathrm{~m}^{-3}\right)\end{array}$ & $\begin{array}{l}\text { Wall } \\
\text { material }\end{array}$ & $\begin{array}{l}\text { Injected } \\
\text { gas }\end{array}$ \\
\hline JET 2001 & Top, single valve & Ohmic, LSN & $\mathrm{D}$ & 2.5 & $\mathrm{CFC}$ & $2.8 \mathrm{~g}\left(\mathrm{C}^{13}\right)$ \\
JET 2004 & OD, multiple valves & H-mode, LSN & $\mathrm{D}$ & 2.6 & $\mathrm{CFC}$ & $9.3 \mathrm{~g}(13 \mathrm{C})$ \\
JET 2007 & OMP, single valve & H-mode, LSN & $\mathrm{D}$ & 3.6 & $\mathrm{CFC}$ & $2.0 \mathrm{~g}(13 \mathrm{C})$ \\
JET 2009 & OD, multiple valves & H-mode, LSN & $\mathrm{D}$ & 4.9 & $\mathrm{CFC}$ & $7.1 \mathrm{~g}(13 \mathrm{C})$ \\
AUG 2003 & OMP, single valve & H-mode, LSN & $\mathrm{H}$ & 8.5 & $\mathrm{C} / \mathrm{W}$ & $0.69 \mathrm{~g}(13 \mathrm{C})$ \\
AUG 2004 & OMP, single valve & H-mode, USN & $\mathrm{H}$ & 9.0 & $\mathrm{C} / \mathrm{W}$ & $0.045 \mathrm{~g}(13 \mathrm{C})$ \\
AUG 2005 & OMP, single valve & L-mode, LSN & $\mathrm{H}$ & 6.0 & $\mathrm{C} / \mathrm{W}$ & $1.1 \mathrm{~g}(13 \mathrm{C})$ \\
AUG 2007 & OMP, single valve & L-mode, LSN & $\mathrm{D}$ & 3.3 & $\mathrm{~W}$ & $0.58 \mathrm{~g}(13 \mathrm{C})$ \\
AUG 2011 & OMP, single valve & L-mode, DN & $\mathrm{H}$ & 5.8 & $\mathrm{~W}$ & $1.0 \mathrm{~g}(13 \mathrm{C})+$ \\
& & & & & & $1.1 \mathrm{~g}(15 \mathrm{~N})$ \\
\hline
\end{tabular}

\section{Review of global tracer-injection experiments in tokamaks}

Tracer-injection experiments have been realized in many different tokamaks, including JET [10,11], AUG [12-15], DIII-D [16-18], JT-60U [19], and TEXTOR [9,20,21] during the past 20 years. AUG, with its tungsten PFCs [22], has provided an ITER- and DEMO-relevant environment for the studies since 2007. The tracer element can be injected into the AUG vessel from a single valve at the outer midplane or at the outer divertor while at JET, injections have also been made from the top of the torus (single valve) and from toroidally periodic configurations of gas valves in the divertor region. Yet, all the JET data are from the era preceding the ITER like wall (ILW), i.e., from a carbon machine and the same holds for experiments carried out at DIII-D. TEXTOR and JT-60U, for their part, are interesting testbeds for elucidating the role of various physical parameters such as the wall material [20] or enhanced re-erosion of the deposited material [23] in the deposition profiles of the impurities.

\subsection{Experimental database from JET and ASDEX Upgrade}

In the global scale, four tracer-injection experiments have been realized at JET $[10,11]$ and five at AUG $[12,13]$. Their most important parameters are summarized in table 1.

Typically, the tracer ${ }^{13} \mathrm{C}$ has been introduced into the vessel in the form of labelled methane $\left({ }^{13} \mathrm{CH}_{4}\right)$ but in the case of the AUG 2011 experiment, both ${ }^{13} \mathrm{CH}_{4}$ and ${ }^{15} \mathrm{~N}_{2}$ were simultaneously injected into the torus. A large parameter space has been covered: The location of the injection source, the type and magnetic configuration of the discharges, the main plasma species and its density, and the amount of gas injected have all been varied. In most cases the injection has been made from a single valve while multiple valves were used in the JET 2004 and 2009 experiments. Having multiple injections will be generally less perturbing for the plasma than a single one. The injection rate was generally kept below $3 \times 10^{21} \mathrm{~s}^{-1}$ since too strong a source would have an impact on the plasma and influence the obtained deposition profiles.

After each experiment, a representative number of first-wall tiles was removed 
from the vessel and the tiles were analyzed using Secondary Ion Mass Spectrometry (SIMS) $[11,12]$ or Nuclear Reaction Analysis (NRA) $[8,15]$. The key results of these analyses can be summarized as follows:

- The resulting deposition patterns are toroidally and poloidally asymmetric. This we notice from the right part of figure 1(a) which shows the average surface densities of ${ }^{13} \mathrm{C}$ on the limiter structures of a Ion Cyclotron Resonance Heating (ICRH) antenna (next to the injection valve) of AUG after the 2011 experiment [13]. These observations set the need for full 3D treatment of impurity migration [24].

- The measured surface densities (in at $/ \mathrm{cm}^{2}$ ) are the highest locally next to the injection valves and typically decay by two orders of magnitude within a distance of a few centimetres. The left part of figure 1(a) illustrates the situation on one poloidal limiter tile of AUG, removed from the vicinity of the impurity source after the 2011 experiment [13]. The edges of the limiter tile in the toroidal direction (at 0 and $200 \mathrm{~mm}$ ) are recessed by about $15 \mathrm{~mm}$ from the central part (100 $\mathrm{mm}$ ) but this seems not to play a major role in the deposition of the impurities: Only next to the injection valve (at $0 \mathrm{~mm}$ ) the surface densities are above $10^{16}-10^{17} \mathrm{at} / \mathrm{cm}^{2}$ (typical values for the main chamber PFCs after the 2011 experiment [13]).

- In the case of main-chamber sources (AUG, JET 2001, and JET 2007), particles migrate towards the inboard side of the vessel and the same holds for a source at the outer divertor (JET 2004, JET 2009) [11,13]. This is attributed to strong flows in the SOL plasma [25].

- Depending on the experiment, 5-50\% of the injected impurity atoms have been observed in surface analyses [11-13]. These numbers have to be taken with caution since they are based on analyses of tiles from a single poloidal cross section and assuming deposition being toroidally symmetric - which does not hold close to the source.

- Based on the JET results in 2009, approximately $30 \%$ of the impurities is directly pumped out from the vessel by cryopumps [11]. Most likely this is an upper limit due to the close proximity of the source (at the outer divertor) and the cryopumps. In addition, samples extracted from tile gaps and remote areas have shown surface densities comparable to those on the plasma-facing surfaces [13].

- Wall material plays a large role for the deposition of the impurities. If the wall is changed from carbon to tungsten, deposition especially at the outer divertor (net-erosion zone) drops by a factor of 10-100 [12]. Similar substrate effect has been observed at TEXTOR [20]. However, if the tungsten PFCs have thick co-deposited layers containing carbon, deuterium, beryllium, and/or boron, the resulting deposition profiles start to resemble those obtained for a carbon wall. This is illustrated in figure $1(\mathrm{~b})$ which shows the deposition profiles of ${ }^{13} \mathrm{C}$ at the divertor of AUG after the 2005 (carbon wall, black curve), 2007 (tungsten wall, red curve), and 2011 (tungsten PFCs with co-deposited layers, blue curve) experiments [12,13]. 

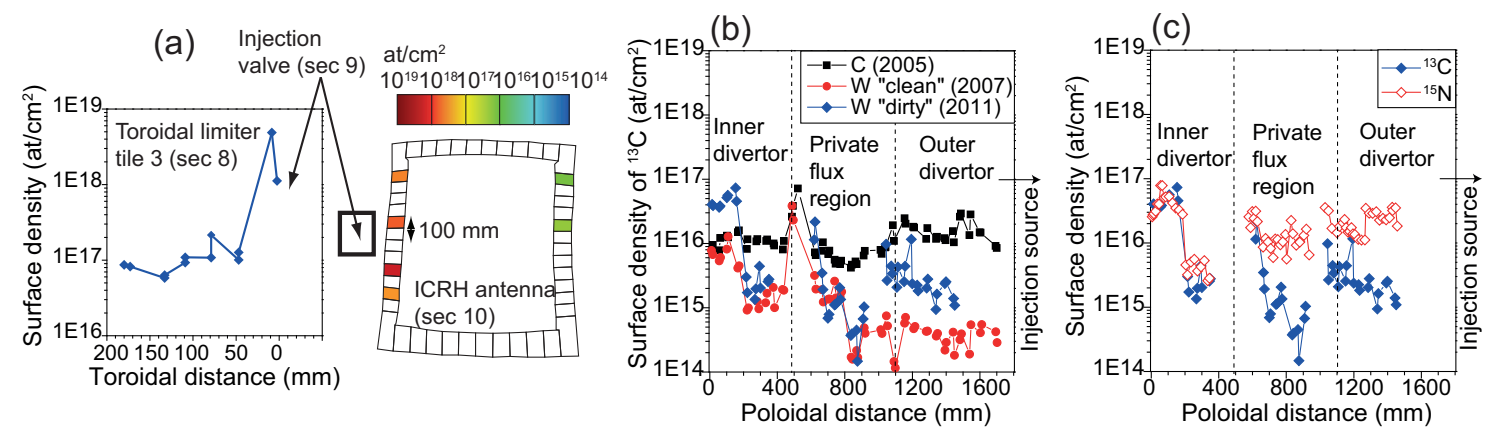

Figure 1. (a) Deposition of ${ }^{13} \mathrm{C}$ on different limiter structures next to the source of the 2011 injection experiment in AUG. (b) Deposition of ${ }^{13} \mathrm{C}$ on PFCs at the divertor of AUG after the 2005 (carbon PFCs), 2007 (clean tungsten PFCs), and 2011 (tungsten PFCs with co-deposited layers) experiments. (c) Comparison between the deposition of ${ }^{13} \mathrm{C}$ and ${ }^{15} \mathrm{~N}$ in the divertor region of AUG in 2011.

- When using ${ }^{15} \mathrm{~N}$ as a tracer [8], the resulting deposition is almost constant throughout the machine while the surface densities of ${ }^{13} \mathrm{C}$ show more variations from region to region, especially a local minimum in the private flux region as illustrated in figure 1(c). The reason for the different deposition of these two elements is attributed to surface chemistry and strong recycling of nitrogen [26].

\subsection{Modelling of the JET and ASDEX Upgrade experiments}

To identify the physical mechanisms that are responsible for producing the experimental deposition profiles discussed above, different edge physics codes have been used to analyze the injection experiments. A proper modelling chain is required such that the output of one code is used as an input for the next one.

The first step is to model the plasma conditions and obtain 2D maps for various parameters such as density, temperature, flow velocity, and electric field in different parts of the vessel. For this, we use either the SOLPS [27] or EDGE2D [28] fluid code. The injected impurity particles are then followed using one of the Monte Carlo codes ERO [29], ASCOT [24], or DIVIMP [30]. Of these, ERO is a 3D code which can be used in different geometries, from limiters to divertors, but simulation volumes are usually spatially limited to keep simulation times manageable. DIVIMP, for its part, is typically used in 2D mode, i.e., toroidal symmetry is assumed. The computational grid of DIVIMP can cover the entire SOL but not the core plasma and only recently the grid has been extended until the walls of the vessel. ASCOT can follow the migration process in the entire tokamak in 3D and in an unrestricted computational domain, even though factors like re-erosion are missing from the present version of ASCOT.

Of the codes presented above, we have used EDGE2D, ERO, and DIVIMP to model the injection experiments at JET $[10,11]$ while in the case of AUG experiments, SOLPS have provided plasma solutions for treating the actual migration process with ERO, ASCOT [24], and DIVIMP [31] 


\section{Understanding the outcomes of the experiments}

In this section, our goal is to understand the experimental results reported in section 2.1 with the help of the modelling tools introduced in section 2.2. The analysis is divided into two parts depending on whether the migrating impurities originate from the outer midplane or from the outer divertor of the tokamak vessel, both strong sources of impurities. In both cases, we want to identify how material is migrating and where it ends up in the torus. In addition, the effect of substrate material and its roughness on the ultimate deposition patterns will be addressed.

\subsection{Migration of material originating from a main-chamber source (ASDEX Upgrade)}

To investigate the migration of impurities released into the plasma at the outer midplane, we take the injection experiment carried out in AUG in 2011 [13] as our starting point. According to table 1 , high-density $\left(n_{\mathrm{e}}=5.8 \times 10^{19} \mathrm{~m}^{-3}\right)$ L-mode discharges in a hydrogen plasma were used. The magnetic configuration showed a secondary separatrix at the top of the vessel, resulting in an ITER-relevant, quasi DN plasma for the migrating impurities. Altogether $9.2 \times 10^{22}$ atoms with a $1: 1$ ratio for ${ }^{13} \mathrm{C}$ and ${ }^{15} \mathrm{~N}$ were puffed into the vessel during the flat-top phases of 11 plasma discharges, corresponding to some $1 \mathrm{~g}$ of each injected isotope, from one valve at the outer midplane, in the toroidal sector 9 (out of 16) of AUG. The plasma current during the shots was $0.8 \mathrm{MA}$, the toroidal magnetic field $-2.5 \mathrm{~T}$, with the grad-B drift of ions pointing towards the divertor, the auxiliary heating power 1.8 MW by neutral beam injection (NBI) and 0.96 MW by electron cyclotron resonance heating $(\mathrm{ECRH})$, and the flat-top time approximately $4.8 \mathrm{~s}$.

The most important experimental observations were strong, localized deposition on limiter structures close to the injection source and large surface densities of the injected ${ }^{13} \mathrm{C}$ at the top of the vessel and on the inner heat shield [13]. Almost 30-35\% of the ${ }^{13} \mathrm{C}$ tracers had been accumulated in the main chamber while only $2-3 \%$ in the divertor region. Compared to the 2007 experiment, where low-density L-mode discharges in deuterium were used, the deposition at the heat shield was 100-1000 times higher in the 2011 configuration as figure 2 illustrates. This can be connected to the different magnetic configurations of the two experiments (LSN in 2007 [12], quasi DN in 2011 [13]). In DN configurations, the plasma becomes almost stagnant at the high-field side while high flow velocities have been measured in the same region for LSN configurations [32]. In addition, the almost two times higher density in 2011 than in 2007 may have shifted the deposition maximum towards the main chamber.

The modelling of the experiment was started by creating plasma solutions with SOLPS. A pure hydrogen plasma was assumed and the upstream electron density at the separatrix, $n_{\mathrm{e}, \mathrm{sep}}$, and the power crossing the core-edge boundary, $P_{\text {core-edge }}$, were varied in the simulations. The resulting profiles for $n_{\mathrm{e}}$ and $T_{\mathrm{e}}$ were fitted to experimental data from Thomson scattering (TS) and reciprocating probe measurements (MEM) at the outer midplane. Outside the SOLPS simulation grid the density and temperature profiles were assumed to decay exponentially as discussed in [33], with decay lengths 


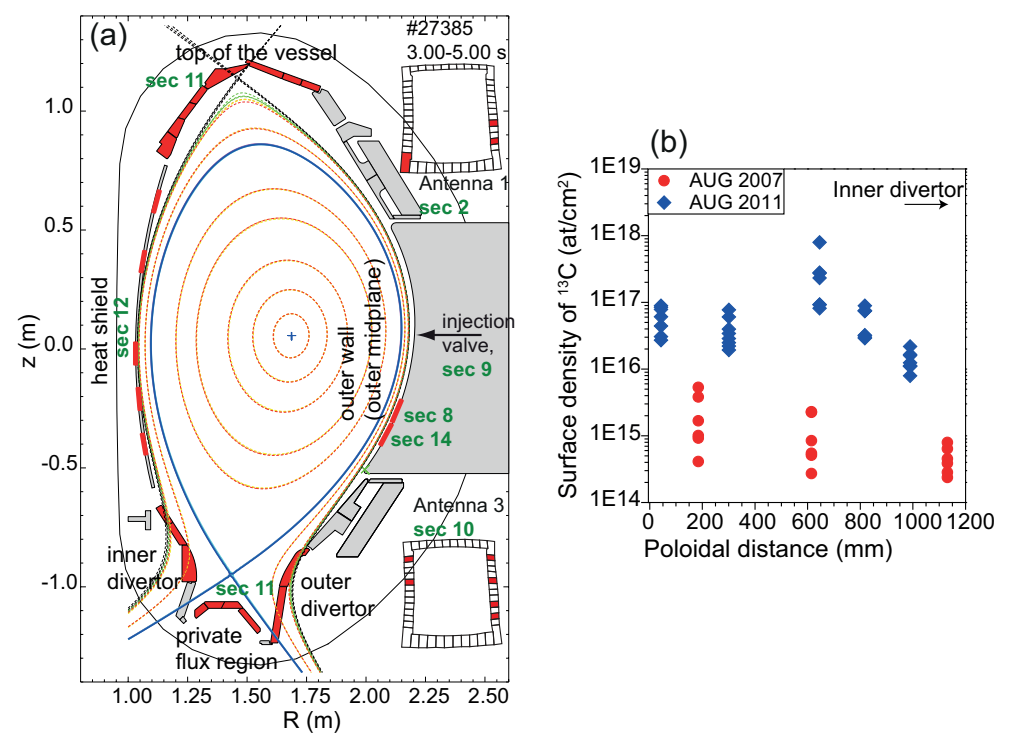

Figure 2. (a) Magnetic configuration during the flat-top phase of one of the discharges (\#27385) of the AUG 2011 experiment. The tiles analyzed after the experiment have been marked in red. (b) Deposition of ${ }^{13} \mathrm{C}$ on the heat-shield region both after the AUG 2011 and 2007 experiments.

based on measured data at the outer midplane. The best fits are shown in figures 3(a) and (b); here $n_{\mathrm{e}, \mathrm{sep}}=2.25 \times 10^{19} \mathrm{~m}^{-3}$ and $P_{\text {core-edge }}=2 \mathrm{MW}$ (denoted by "basecase" in ERO and ASCOT simulations). In the divertor region, the simulated $n_{\mathrm{e}}$ and $T_{\mathrm{e}}$ profiles deviated from the measured ones by a factor of two, which is a typical problem for fluid codes, especially in high-density plasmas [34]. Furthermore, the simulations predicted rather weak plasma flow in the main chamber (see figure 3(c)), which is in contradiction with the existing experimental data on SOL flows [35]. Therefore, an experimentally motivated profile with a Mach 0.5 flow towards the inner divertor and a stagnation point between the X-point and the outer midplane was imposed on top of the SOLPS plasma solution as shown in figure 3(c); below the stagnation point the flow is towards the outer divertor. Even though the solution is not self-consistent anymore, the imposed flow profile is more realistic to be included in the ERO and ASCOT simulations. The applied profile does not take into account variations in the poloidal or radial directions due to the lack of experimental data for the plasmas studied here. Some approaches have been made to reproduce the observed strong SOL flows with SOLPS (see, e.g., [36]) but they have not yet been implemented in these studies.

The incoming ${ }^{13} \mathrm{CH}_{4}$ and ${ }^{15} \mathrm{~N}_{2}$ molecules were traced by ERO until they were dissociated and finally ionized in the SOL. The simulations were carried out in several different SOLPS backgrounds and using the magnetic equilibrium from one of the discharges (\#27385, see figure 2(a)) of the AUG 2011 experiment. The simulation volume, schematically shown in figure 4 (a), had dimensions $\Delta R=1.7 \mathrm{~m}$ and $\Delta z=1.4 \mathrm{~m}$ and assumed to be toroidally symmetric. The molecules were assumed to obey Maxwellian energy distribution with a mean energy of $0.05 \mathrm{eV}$. The methane breakup 
(a)

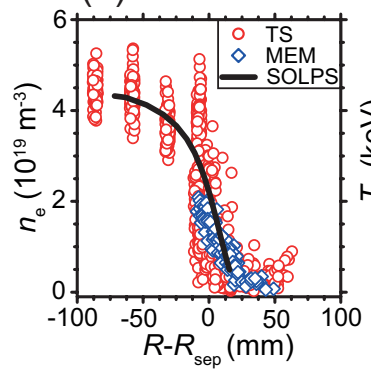

(b)

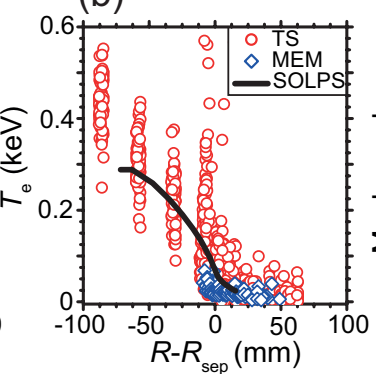

(c)

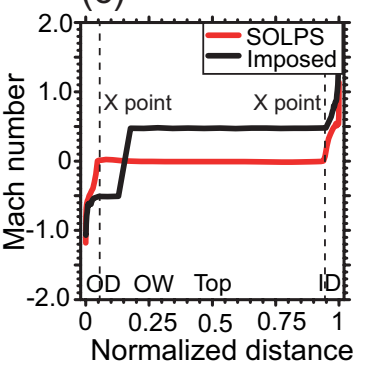

Figure 3. (a,b) Experimental (TS and MEM) and simulated (SOLPS) profiles of (a) $n_{\mathrm{e}}$ and (b) $T_{\mathrm{e}}$ at the OMP during the AUG 2011 experiment. (c) SOLPS prediction (red) for the poloidal flow profile of the plasma in the SOL together with the imposed flow profile (black) used in ERO and ASCOT modelling. The poloidal locations of the $\mathrm{X}$ point at the outer $(0.055)$ and inner (0.949) sides of the vessel are denoted by dashed lines. Here, $\mathrm{ID}=$ inner divertor, $\mathrm{OD}=$ outer divertor, and $\mathrm{OW}=$ outer wall.

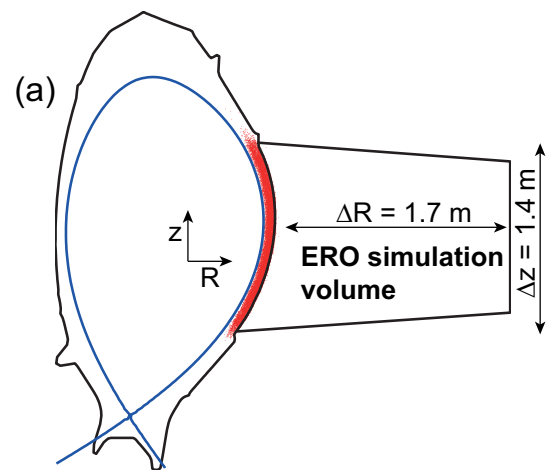

(b)

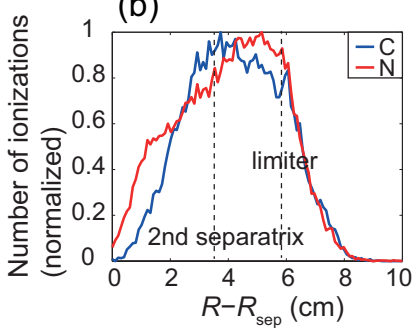

(c)

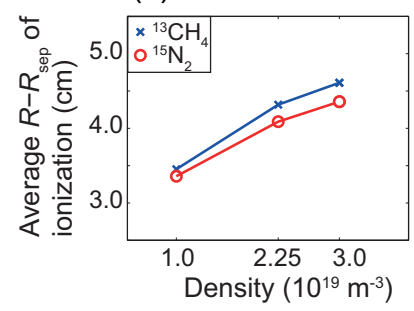

Figure 4. (a) Schematic illustration of the ERO simulation box for the AUG 2011 experiment. (b) ERO results for the radial profiles of ${ }^{13} \mathrm{C}^{+}$and ${ }^{15} \mathrm{~N}^{+}$ions in the SOL when using the basecase SOLPS plasma with the imposed flow. The flux surface of the secondary separatrix $(3.6 \mathrm{~cm})$ and the position of the limiter surface $(5.9 \mathrm{~cm})$ have been marked in the figure. (c) Average distance of the ionization cloud from the separatrix as a function of density.

was modelled according to the discussion in [37]. In the case of nitrogen, ERO was upgraded by implementing the break-up chain of $\mathrm{N}_{2}$ into it [38-41] and assuming a total kinetic energy of $2.0 \mathrm{eV}$ for the decay products.

The radial profiles of the resulting ionization clouds are relatively homogeneous with the main peak in the far SOL, a few centimeters away from the separatrix, and rather similar for both ${ }^{13} \mathrm{C}^{+}$and ${ }^{15} \mathrm{~N}^{+}$as one notices from figure $4(\mathrm{~b})$; here the basecase SOLPS plasma with the imposed flow has been used. When increasing the plasma density, the ions are born closer to the wall. This is illustrated in figure 4(b) where the average ionization distance from the separatrix as a function of density is shown. A similar but much weaker effect was noticed when changing the power (from $1 \mathrm{MW}$ to $3 \mathrm{MW})$. 
The ionization profiles were used as inputs for ASCOT. In addition, a realistic 3D wall geometry of the AUG torus was constructed [24] and the anomalous diffusion coefficient of the impurities was scanned from $0.25 \mathrm{~m}^{2} / \mathrm{s}$ (basecase) to $1.0 \mathrm{~m}^{2} / \mathrm{s}$. The simulations were able to explain the highly localized deposition on the limiter structures at the outer wall as well as the strong inventories of impurity atoms at the top of the vessel. Figure 5(a)) shows the resulting 2D deposition pattern for the basecase plasma (see figure 3) with the imposed flow profile. Notice that, excluding the outer midplane, the deposition profiles are rather uniform toroidally, mainly because of strong magnetic shear and the toroidally symmetric wall geometry of AUG [24]. The plasma flow seems to be play a significant role in transporting impurities to different regions of the torus as indicated by the deposition pattern following the direction of the magnetic field lines in figure 5(a). According to figure 5(b), using the weak SOLPS flow (blue bars) from figure 3(c) would result in almost $50 \%$ of the injected impurities being deposited at the outer divertor while with the imposed flow (red bars) this fraction drops to less than $5 \%$ - resulting in good agreement with experimental results in this part of the torus (yellow bars). A key factor seems to be that a sufficiently strong flow is established at the low-field side to drive particles to the high-field side.

However, the ratio between deposition on the heat shield and on the inner divertor is almost the opposite to what has been experimentally observed. This can be due to the DN magnetic configuration as we discussed above. In fact, under DN conditions the SOL plasma is split into two parts: One at the low-field side, the other one at the highfield side. When using the imposed flow at the low-field side and the almost stagnant SOLPS flow at the high-field side (a situation observed, e.g., at Alcator C-Mod [32]), we observed more particles being deposited on the heat shield and fewer on the inner divertor than in the basecase. The shape of the secondary separatrix (see figure 2(a)) may provide an explanation to this behavior: While the secondary separatrix is $3.6 \mathrm{~cm}$ away from the primary one and $2.3 \mathrm{~cm}$ from the limiter surfaces at the low-field side (recall figure 4(b)), it almost touches the inner wall at the high-field side. Now if the flow becomes very weak at the high-field side, the particles are deposited already on the heat shield before reaching the divertor region. The effect becomes ever more pronounced as the plasma density increases (see figure $5(\mathrm{c})$ ). The reason is that in denser plasmas the ionization profile peaks closer to the outer wall and, due to a stronger coupling to the plasma flow, the transport of the resulting particles is more strongly towards the main-chamber walls - instead of the divertor surfaces. A similar situation results when increasing radial diffusion of the impurities, as shown in figure $5(\mathrm{~d})$.

However, over long time scales, surface compositions of PFCs will be modified as a result of material being re-eroded and re-deposited in multiple steps. This will result in walking of the particles along the PFCs into new locations [10]. Also re-erosion during the start-up and termination phases of the discharges may alter the primary deposition profiles. Stepwise migration of impurities has been observed to play a large role, e.g., in DIVIMP [3] and WallDYN [42] modelling of the transport of beryllium in the ILW configuration of JET. The present version of ASCOT does not include re- 


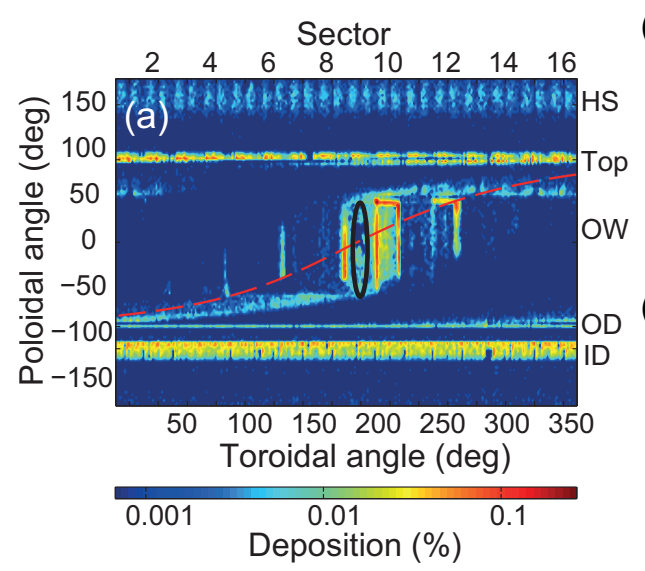

(b)

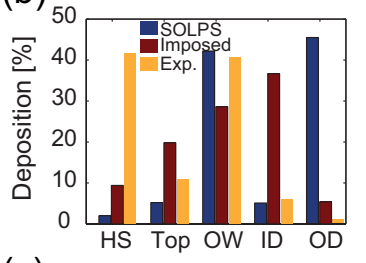

(c)

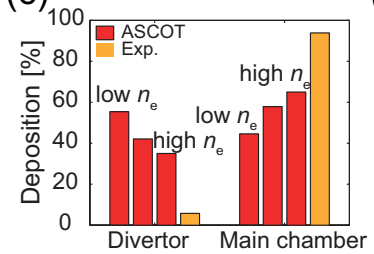

(d)

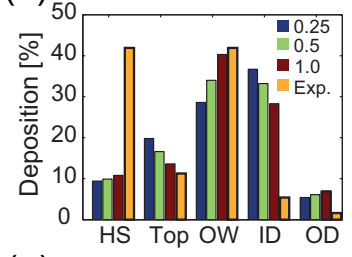

$(\mathrm{e})$

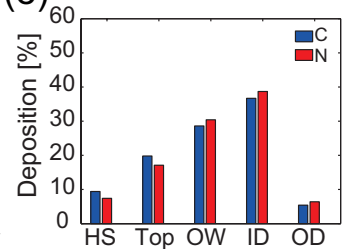

Figure 5. (a) ASCOT prediction for the $2 \mathrm{D}$ deposition profile of ${ }^{13} \mathrm{C}$ impurities after the AUG 2011 experiment when using the basecase SOLPS plasma with the imposed flow. The black ellipse marks the source and the red dashed line the direction of the magnetic field. (b) Effect of SOL flow profile on the deposition of ${ }^{13} \mathrm{C}$ in different regions of AUG. Blue bars correspond to using the weak SOLPS flow, red bars to the imposed-flow case, and yellow bars to experimental data (percentages of the ${ }^{13} \mathrm{C}$ found). (c,d) Effect of (c) density and (d) anomalous diffusion coefficient $\left(D_{\perp}\right.$, in $\mathrm{m}^{2} / \mathrm{s}$ ) on the deposition of ${ }^{13} \mathrm{C}$ in different regions of $\mathrm{AUG}$ together with experimental results. (e) Comparison between the calculated deposition of ${ }^{13} \mathrm{C}$ and ${ }^{15} \mathrm{~N}$ impurities in AUG. Here, $\mathrm{HS}=$ heat shield, $\mathrm{OW}=$ outer wall, $\mathrm{ID}=$ inner divertor, and $\mathrm{OD}=$ outer divertor.

erosion so future work could include combining the 3D features of ASCOT with the surface dynamics described by WallDYN.

The discussion above has concentrated on plasma physics while the qualitatively different measured deposition profiles of ${ }^{13} \mathrm{C}$ and ${ }^{15} \mathrm{~N}$ require taking surface effects into account. According to ASCOT simulations (see figure 5(e)), the deposition of these two isotopes should be almost identical (blue and red bars) but experimentally ${ }^{15} \mathrm{~N}$ shows a uniform deposition profile throughout the torus, even in the private flux region where almost no ${ }^{13} \mathrm{C}$ has been observed (figure $1(\mathrm{c})$ ). This could be explained by the fact that nitrogen concentrations on a $\mathrm{W}$ surface quickly saturate and any excess nitrogen is degassed [26]. Nitrogen thus becomes a recycling isotope in the torus, contrary to the case of carbon or beryllium.

\subsection{Migration of material originating from a divertor source (JET)}

To investigate the physical mechanisms related to transport of impurities born in the divertor region, we concentrate on the experiment carried out at JET in 2009 [11]. Altogether, $3.3 \times 10^{23}$ atoms or $7.1 \mathrm{~g}$ of ${ }^{13} \mathrm{C}$ were injected into the SOL plasma close to the outer strike point from 24 valves, located toroidally periodically next to the corresponding tiles 5 (see figure 6(a)). The experiment consisted of 30 identical, ELMy H-mode discharges with the LSN configuration in a deuterium plasma (density 

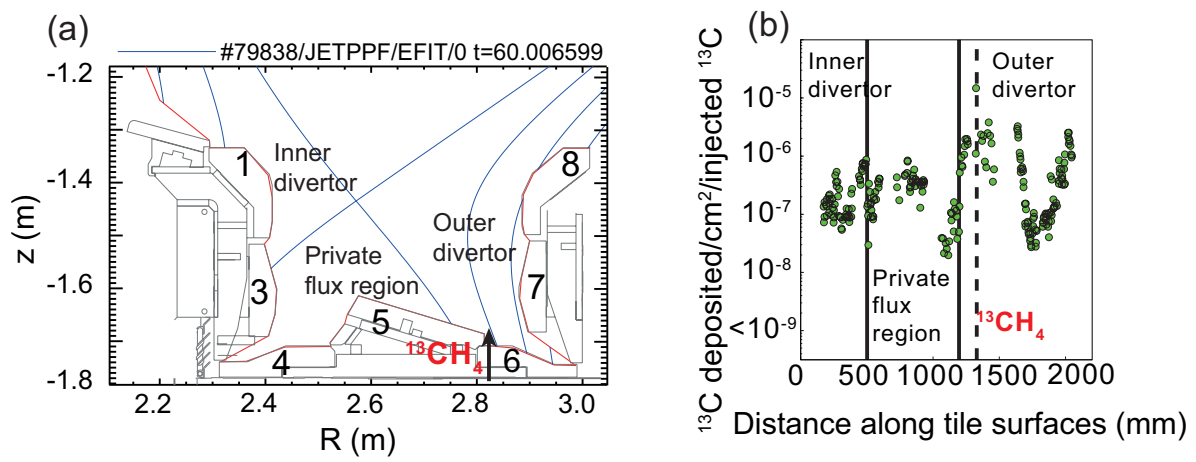

Figure 6. (a) Magnetic configuration of one of the discharges (\#79838) of the JET 2009 experiment. Also the geometry of the divertor and the location of the puffing valve are shown. (b) Toroidally averaged poloidal deposition profile of ${ }^{13} \mathrm{C}$ (per injected ${ }^{13} \mathrm{C}$ atoms) on the divertor tiles after the 2009 experiment.

$\left.n_{\mathrm{e}}=5.0 \times 10^{19} \mathrm{~m}^{-3}\right)$. The operational scenario was representative of a typical discharge during the 2008-2009 campaign. The plasma current was 2.5 MA, the toroidal magnetic field $2.5 \mathrm{~T}$, the auxiliary heating power $15 \mathrm{MW}$ of NBI, and the injection took place during $6 \mathrm{~s}$ of the flat-top phase.

Post mortem analyses [11] showed a strong toroidal deposition band near each injection valve, with surface density fractions up to $1.5 \times 10^{-5}$ at $/ \mathrm{cm}^{2}$ per injected ${ }^{13} \mathrm{C}$ atom [43]. In the outer parts of the divertor, these fractions varied between $5 \times 10^{-8}$ and $5 \times 10^{-6}$ while in the inner divertor the deposition profile was more homogeneous as we see from figure 6(b). By assuming toroidally symmetric deposition, approximately $13 \%$ of the injected atoms had been accumulated in the outer divertor and less than $2 \%$ in the inner divertor. The latter number is, however, still large compared to the $0.4 \%$ deposition in the main chamber.

To simulate the deposition profile in figure 6(b), plasma solutions were created using EDGE2D. The main input parameter was the power entering the grid boundary, here approximately $10 \mathrm{MW}$, and the focus was on the steady-state, inter-ELM phase of the discharges. Exponentially decaying profiles for density and temperature were used outside the simulation grid. The best fits to the experimental profiles of $n_{\mathrm{e}}, T_{\mathrm{e}}, n_{\mathrm{i}}$, and $T_{\mathrm{i}}$ at the outer midplane and at the outer divertor correspond to $n_{\mathrm{e}, \mathrm{sep}}=2.67 \times 10^{19} \mathrm{~m}^{-3}$. The poloidal flow profile qualitatively agrees with the measurement data - over the top of the vessel towards the inner divertor — but the Mach values in the main chamber are too low: Around Mach 0.2 while experimentally they should be closer to Mach 0.5 [35]. This was not surprising since several factors, especially the $\mathbf{E} \times \mathbf{B}$ drift [34], affecting the plasma flow are missing in the present version of EDGE2D. Therefore, the simulations were re-run using an artificial momentum source at the outboard side of the vessel, corresponding to Mach 0.5. Such an imposed flow results in redistribution of the plasma flux in the main chamber and, as a result, in negligible effects (less than 50\%) on the density and temperature in the divertor region. 
The EDGE2D solutions with the imposed flow were then used in subsequent ERO simulations for methane breakup and carbon migration. The simulation volume covered the entire lower divertor of JET as illustrated in figure 7(a)). The particles that exited the simulation volume were either re-introduced into the box or considered as lost. The re-introduction was performed by following the particles in the main-chamber SOL and core-edge boundary using DIVIMP. The underlying assumption was that the particles re-entering the simulation box had been thermalized in collisions with the background ions. The anomalous diffusion coefficient of carbon ions was set to $D_{\perp}=1.0 \mathrm{~m}^{2} / \mathrm{s}$. This value describes best the situation at JET while previous modelling on AUG has found best agreement with the experimental results with $D_{\perp}=0.25 \mathrm{~m}^{2} / \mathrm{s}$. The reflection and sticking of the tracers was treated similarly to the case in [43], in particular the erosion of the deposited layers was assumed to be enhanced by a factor of 5 , as motivated in [23].

The simulations could qualitatively explain the strong deposition peak close to the injection valve as well as the decreasing tendency of deposition when moving away from the source along the outer-divertor PFCs. This can be seen in figure 7(b) where the toroidally averaged deposition profiles for ${ }^{13} \mathrm{C}$ are shown for different simulation cases. The deviations between the experimental and simulated profiles - mainly the abrupt drop in deposition in the border zone between the divertor tiles 7 and 8 - is attributed to the wall contour used in ERO (violet curve in figure $7(\mathrm{a})$ ) being slightly different from the real wall (blue curve). Based on our simulations, even such subtle differences can lead to largely different migration behaviour of the impurities.

ERO identified several mechanisms which transport particles from the outboard to the inboard side of the vessel. These become evident from the three different innerdivertor profiles in figure 7(b). In case A, transport through the main SOL and the core is turned off, resulting in more than two orders of magnitude too low deposition at the inner divertor and in the private flux region - although re-erosion and crossfield drifts are included in the simulations. Case B represents the primary deposition pattern including the transport term via the main chamber (both main SOL and core) but excluding re-erosion. Finally, in case $\mathrm{C}$, both transport and re-erosion are activated resulting in many more particles in the private flux region than in case B.

From these profiles we can conclude that impurities are driven by SOL flows over the top of the machine or through the core to the inner divertor. The qualitative picture is therefore similar to the case of a main-chamber source discussed in section 3.1. As a result of re-erosion, the impurities ending up at the inner divertor walk downwards along the PFCs [10], finally across the separatrix into the private flux region. In contrast, at the outboard side re-erosion has no noticeable effects on the deposition profiles of impurities beacuse of particles walking away from the outer strike point and the source.

The importance of re-erosion in determining the final deposition profiles is evidenced by the data extracted from TEXTOR experiments and related ERO modelling [9] as well as from spectroscopic investigations during the ILW period of JET [3], as already discussed in section 3.1. Re-erosion could be even stronger if ELMs or transients like disruptions were included in the simulations [44]. In general, clarifying the role of 


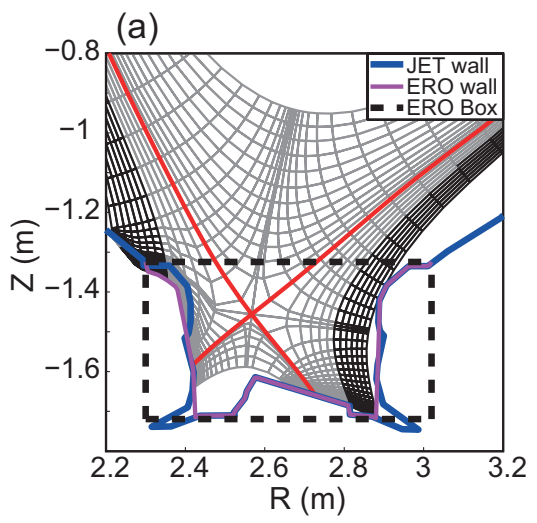

(b)

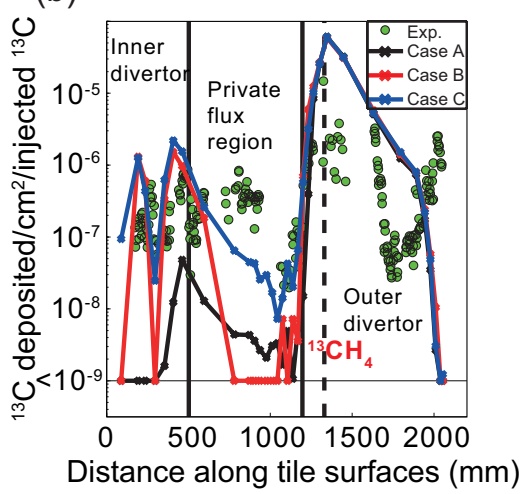

Figure 7. (a) Calculation grid used in ERO and DIVIMP simulations of the JET 2009 experiment together with the simulation box of ERO and the contour of the wall used as an input in ERO. (b) Simulation results together with experimental data from figure 6(b) for three cases. Case $\mathrm{A}=$ no transport, Case $\mathrm{B}=$ transport included, Case $\mathrm{C}=$ transport and re-erosion turned on.

transients requires more investigations in the future.

In principle, the particles could be dragged from the outer to the inner side of the divertor via the private flux region by $\mathbf{E} \times \mathbf{B}$ driven plasma flows. However, this kind of shortcut is not seen in our simulations: Hardly any particle enters the private plasma region across the outer strike point. Experimentally, this is evidenced by a sharp drop in the measured ${ }^{13} \mathrm{C}$ surface densities inboard of the outer strike point.

\subsection{Effect of surface material on the final deposition patterns}

In addition to the discussion above, the material properties of PFCs have to be included in the full explanation of the deposition patterns of the tracer elements. For example, deposition on tungsten is 2-3 orders of magnitude smaller than that on carbon as we noticed in section 2.1. This result, however, holds only for smooth surfaces with surface roughness of $R_{\mathrm{a}}<1 \mu \mathrm{m}$. As the roughness increases, deposition profiles on $\mathrm{W}$ and on $\mathrm{C}$ start to resemble each other. This can be observed in figure 8(a) where the deposition of ${ }^{13} \mathrm{C}$ on the relatively rough $\left(R_{\mathrm{a}}=5-10 \mu \mathrm{m}\right)$ tungsten and carbon surfaces at the outer divertor of JET in 2009 is shown. The same conclusion has been drawn in TEXTOR [19]. In contrast, when the $\mathrm{W}$ surface has a clear co-deposited layer the roughness effect practically disappears. This we notice from figure 8(b) where the deposition profiles of ${ }^{13} \mathrm{C}$ on $\mathrm{W}$-coated marker stripes with different surface roughness in the outer strike-point region of AUG have been reproduced (AUG 2011 experiment). The marker stripes were poloidally oriented, toroidally next to each other, and the thickness of their $\mathrm{W}$ coatings was $1.5-2 \mu \mathrm{m}$. Before plasma exposure, their surface roughnesses were $R_{\mathrm{a}}=5.5 \mu \mathrm{m}$ (rough), $R_{\mathrm{a}}=1.5 \mu \mathrm{m}$ (nominal), $R_{\mathrm{a}}=0.8 \mu \mathrm{m}$ (medium), and $R_{\mathrm{a}}=0.4 \mu \mathrm{m}$ (smooth). Up to 1- $\mu \mathrm{m}$ thick boron-rich surface layers have led to smoothening of the surfaces, which have made them almost equal for the incoming ${ }^{13} \mathrm{C}$ particles. In addition, co-deposited 


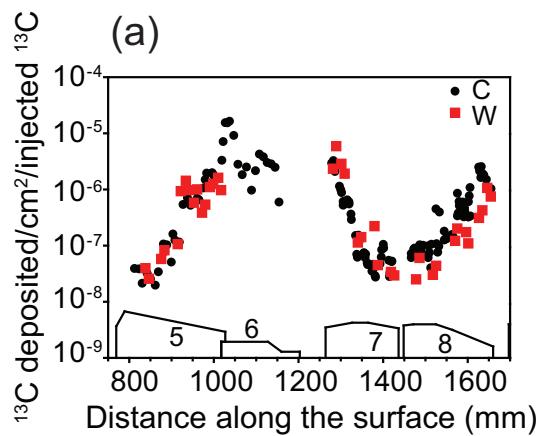

(b)

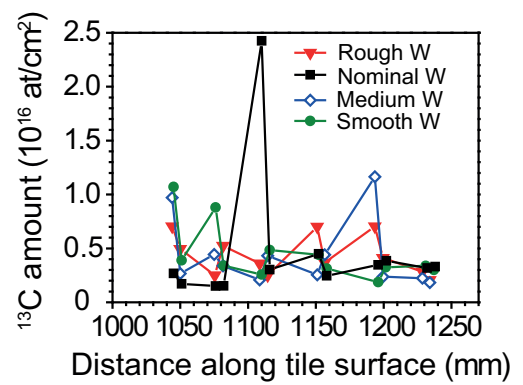

Figure 8. (a) Poloidal deposition profiles of ${ }^{13} \mathrm{C}$ on carbon and tungsten on the divertor tiles of JET after the 2009 experiment. (b) Poloidal deposition profiles of ${ }^{13} \mathrm{C}$ on different marker stripes of the outer strike point tiles of AUG after the 2011 experiment.

layers generally enhance deposition (see figure 1(b)).

\section{Conclusions}

This article has concentrated on the global migration of impurities at JET and AUG. To this end, tracer elements have been injected into the vessel during dedicated plasma discharges since 2001. Surface analyses have shown that the deposition profiles are generally not toroidally symmetric but a distinct 3D pattern is formed on protruding surface features. Nonetheless, in certain locations of the torus, e.g., in the divertor in the case of a main-chamber source, toroidal symmetry is still a reasonable assumption.

Several factors have been observed to affect the transport of particles, the most important of them being flows in the SOL plasma, magnetic configuration and local plasma conditions ( $\mathrm{such}$ as $n_{\mathrm{e}}$ and $T_{\mathrm{e}}$ ), proximity of the wall, and re-erosion. Generally, the flows drive particles from the outboard towards the inboard side of the tokamak, either through the top of the vessel or via the core plasma. Unfortunately, such flow profiles have not been reproduced in the simulations but one has been forced to impose them on top of background plasma solutions. Re-erosion, for its part, has been observed to considerably modify the primary deposition profiles, as a result of stepwise migration of impurities along the PFCs. The process is particularly important in the divertor region but the JET ILW data indicate that re-erosion should also be taken into account in the main chamber. All the deposition patterns are further modified by ELMs and other transient effects; none of them have yet been included in these studies.

Deposition of the impurities is also affected by surface chemistry. This is evidenced by the qualitatively different deposition profiles of nitrogen and carbon on PFCs. Moreover, the rougher the surface is and the thicker co-deposited layers it contains, the stronger is the deposition on it. Material is not only accumulated on plasmafacing surfaces but shadowed areas, such as gaps between PFCs and remote parts of the tokamak vessel, typically contain significant impurity inventories. These may be 
the final destinations for all the impurities being eroded from the plasma-facing surfaces over long time scales as evidenced by our data from AUG [13].

As a conclusion, we can qualitatively explain the final deposition profiles of migrating impurities but to predict transport of particles in real fusion reactors, the simulation codes have to have a proper background plasma and transients, surface chemistry, and re-erosion chains have to be all included in the simulation codes. The models have also to be verified in an ITER-relevant environment, and for this the ILW at JET provides a unique opportunity.

\section{Acknowledgements}

This work, supported by the European Communities under the contract of Association between Euratom and Tekes, was carried out within the framework of the European Fusion Development Agreement. The views and opinions expressed herein do not necessarily reflect those of the European Commission.

\section{References}

[1] Pitts R A et al 2005 Plasma Phys. Control. Fusion 47 B303

[2] Roth J et al 2008 Plasma Phys. Control. Fusion 50103001

[3] Krieger K et al 2013 J. Nucl. Mater. 438 S262

[4] Dux R et al 2011 Nucl. Fusion 51053002

[5] Mayer M et al 2009 Phys. Scr. T138 014039

[6] Coad J P et al 2011 Phys. Scr. T145 014003

[7] Stangeby P C 2011 J. Nucl. Mater. 415 S278

[8] Petersson P et al 2013 J. Nucl. Mater. 438 S616

[9] Kirschner A et al 2011 J. Nucl. Mater. 415 S239

[10] Strachan J D et al 2008 Nucl. Fusion 48105002

[11] Likonen J et al 2011 Phys. Scr. T145 014004

[12] Hakola A et al 2010 Plasma Phys. Control. Fusion 52065006

[13] Hakola A et al 2013 J. Nucl. Mater. 438 S694

[14] Pugno R et al 2009 J. Nucl. Mater. 390-391 68

[15] Aho-Mantila L et al 2012 Nucl. Fusion 52103007

[16] Groth M et al 2007 Phys. Plasmas 14056120

[17] Wampler W R et al 2007 J. Nucl. Mater. 363-365 72

[18] Elder J D et al 2011 J. Nucl. Mater. 415 S513

[19] Ueda Y et al 2009 Nucl. Fusion 49065027

[20] Kreter A et al 2008 Plasma Phys. Control. Fusion 50095008

[21] Brezinsek S et al 2009 Phys. Scr. T138 014022

[22] Neu R et al 2009 Phys. Scr. T138 014038

[23] Kirschner A et al 2013 J. Nucl. Mater. 438 S723

[24] Miettunen J et al 2012 Nucl. Fusion 52032001

[25] Makkonen T et al 2013 J. Nucl. Mater. 438 S410

[26] Schmid K et al 2010 Nucl. Fusion 50025006

[27] Schneider R et al 2006 Contrib. Plasma Phys. 463

[28] Taroni A et al 1992 Contrib. Plasma Phys. 32438

[29] Kirschner A et al 2000 Nucl. Fusion 40989

[30] Stangeby P C et al 1988 Nucl. Fusion 281945 
[31] Makkonen T et al 2011 J. Nucl. Mater. 415 S479

[32] LaBombard B et al 2004 Nucl. Fusion 441047

[33] Miettunen J et al 2013 Proc. 40th EPS Conf. on Plasma Physics (Espoo, Finland) P2.125

[34] Wischmeier M et al 2011 J. Nucl. Mater. 415 S523

[35] Lipschultz B et al 2007 Nucl. Fusion 471189

[36] Coster D P et al 2004 Proc. 20th IAEA Fusion Energy Conference (Vilamoura, Portugal) IAEA$\mathrm{CN}-116 / \mathrm{TH} / \mathrm{P} 5-18$

[37] Janev R and Reiter D 2002 Collision Processes of Hydrocarbon Species in Hydrogen Plasmas: l. The Methane Family, Report Jül-3966, ISSN 0944-2952

[38] Rapp D et al 1965 J. Chem. Phys. 431464

[39] Cosby P C 1993 J. Chem. Phys. 989544

[40] Bahati E M et al 2001 J. Phys. B: At. Mol. Opt. Phys. 342963

[41] Brook E et al 1978 J. Phys. B: : At. Mol. Opt. Phys. 113115

[42] Schmid K 2013 J. Nucl. Mater. 438 S484

[43] Likonen J et al 2013 J. Nucl. Mater. 438 S762

[44] Airila M et al 2013 Proc. 40th EPS Conf. on Plasma Physics (Espoo, Finland) P2.126

[45] Ueda Y et al 2009 J. Nucl. Mater. 390-391 44 